\title{
Norcantharidin enhances ABT-263-mediated anticancer activity in neuroblastoma cells by upregulation of Noxa
}

\author{
XIAOHUI WANG ${ }^{1}$, ZHIMIN GU $^{2}$, GONGQUAN $\mathrm{LI}^{3}$, SHUFENG ZHANG ${ }^{1}$, \\ ZHENJIE CAO $^{4}$, ZHANFENG YANG ${ }^{5}$ and GUANGZHI LIU ${ }^{6}$
}

\begin{abstract}
${ }^{1}$ Department of Pediatric Surgery, People's Hospital of Zhengzhou University; ${ }^{2}$ Department of Ophthalmology, The Fifth Affiliated Hospital of Zhengzhou University; ${ }^{3}$ Department of Hepatobiliary and Pancreatic Surgery, The First Affiliated Hospital of Zhengzhou University; ${ }^{4}$ Department of Pediatric Surgery, The Third Affiliated Hospital of Zhengzhou University;

${ }^{5}$ Department of Hepatobiliary Surgery, The Fifth Affiliated Hospital of Zhengzhou University; ${ }^{6}$ Department of Obstetrics and Gynecology, People's Hospital of Zhengzhou University, Zhengzhou, Henan 450003, P.R. China
\end{abstract}

Received March 17, 2014; Accepted April 16, 2014

DOI: 10.3892/or.2014.3228

\begin{abstract}
Neuroblastoma is an aggressive childhood disease. Even with intensive conventional treatments, the long term survival rate for children with neuroblastoma remains less than $40 \%$, highlighting the importance of finding new therapies. Bcl-2 family proteins play crucial roles in survival, proliferation and chemotherapeutic resistance of neuroblastoma cells. Therefore, targeting Bcl-2 with small molecule inhibitor ABT-263 could be a novel strategy for treatment of neuroblastoma. However, previous studies indicated that most neuroblastoma cell lines are resistant to ABT-263-mediated apoptosis. Thus, it is crucial to discover approaches that could overcome ABT-263 resistance. In this study, we examined the anticancer activity of ABT-263 in combination with norcantharidin (NCTD), a small-molecule anticancer drug derived from a traditional Chinese medicine, in human malignant neuroblastoma cells. We found that NCTD substantially enhanced ABT-263-mediated apoptosis induction, cell viability inhibition, and clonal formation inhibition in neuroblastoma SH-SY5Y and CHLA-119 cell lines. Moreover, the combination anticancer activity was accompanied by upregulation of Noxa, and was associated with characteristics of mitochondrial apoptosis signaling, such as cytosolic release of cytochrome $c$, activation of caspase- $9,-3$, and cleavage of PARP. Notably, we observed that knockdown of Noxa significantly attenuated cell death induction by cotreatment with ABT-263 and NCTD,
\end{abstract}

Correspondence to: Dr Xiaohui Wang, Department of Pediatric Surgery, People's Hospital of Zhengzhou University, 7 Weiwu Road, Jinshui, Zhengzhou, Henan 450003, P.R. China

E-mail: xhwanggql@gmail.com

Professor Guangzhi Liu, Department of Obstetrics and Gynecology, People's Hospital of Zhengzhou University, 7 Weiwu Road, Jinshui, Zhengzhou, Henan 450003, P.R. China

E-mail: guangzhi72@126.com

Key words: neuroblastoma, ABT-263, norcantharidin, Noxa indicating Noxa essentially contributes to the combination anticancer effect. Collectively, our study demonstrated that NCTD could overcome ABT-263-resistance in neuroblastoma cells, and suggested that combinational treatment of ABT-263 with NCTD might be a novel therapeutic option for children with neuroblastoma.

\section{Introduction}

Neuroblastoma is the most common extracranial solid tumor of children and accounts for $15 \%$ of pediatric cancer deaths $(1,2)$. Neuroblastoma may regress spontaneously in children aged $<18$ months; however, neuroblastoma in children $>18$ months is always associated with aggressive characteristics and is considered as a high-risk disease. Even with intensive combination treatment with surgery, chemotherapy and radiation, the long term survival rate for patients with neuroblastoma remains $<40 \%$, highlighting the necessity to explore novel therapies for this devastating childhood disease (1-3).

Apoptosis is the process of programmed cell death that plays a key role in maintenance of cell homeostasis and differentiation. Resistance of neuroblastoma cells to apoptosis is a major contributing factor to the aggressive nature of the highrisk neuroblastoma (2-4). Among many mechanisms that cause resistance of neuroblastoma cells to apoptosis, elevated expression of antiapoptotic members of Bcl-2 family proteins such as $\mathrm{Bcl}-2, \mathrm{Bcl}-\mathrm{xL}$, and $\mathrm{Mcl}-1$ has attracted particular attention, since these proteins negatively regulate mitochondria-mediated apoptosis (5). It was reported that $>50 \%$ of neuroblastoma tumor tissues and the majority of neuroblastoma cell lines express a high level of Bcl-2 (4,6). Moreover, overexpression of $\mathrm{Bcl}-2$ protein was found to be closely associated with poor response to apoptosis induction by conventional therapies such as radiotherapy and chemotherapy (7-9). Hence, restoration of apoptosis by targeting Bcl-2 might be a promising strategy in managing neuroblastoma.

It has been proposed that small-molecule $\mathrm{Bcl}-2$ inhibitors ABT-737 and ABT-263 treat human cancer $(10,11)$. Preclinical studies showed that ABT-737 and ABT-263 are able to potently induce apoptosis and exhibit strong anticancer 
activity in certain types of cancer through inhibition of Bcl-2 and Bcl-xL (10,11). In particular, clinical trials with ABT-263 have shown promising anticancer activity in hematologic tumors and lung cancer, demonstrating ABT-263 is a promising anticancer drug $(12,13)$. Nevertheless, a fundamental problem of ABT-737 and ABT-263 is that the majority of solid tumors, including neuroblastoma, are resistant to the apoptosis induction by these two new drugs, posing a chief problem for the potential utility in management of these cancers (14). Thus, major efforts have focused on identifying approaches that could overcome ABT-737/ABT-263-resistance through combinational strategies (15-17).

Norcantharidin (NCTD) is a small-molecule anticancer drug derived from a traditional Chinese medicine blister beetle (18-20). We recently reported that NCTD could overcome the resistance to ABT-737 in a panel of hepatocellular cancer cell lines (21). In the current study, we investigated the anticancer activity of ABT-263 in combination with NCTD in neuroblastoma SH-SY5Y and CHLA-119 cell lines. We found that NCTD markedly increased the expression of Noxa, an endogenous Mcl-1 inhibitor, which leads to significant enhancement of ABT-263-mediated anticancer activity in these neuroblastoma cells. Since NCTD is routinely used to treat patients with cancer in clinic, our findings have translational significance in managing neuroblastoma.

\section{Materials and methods}

Cell lines and compound preparations. Neuroblastoma cell lines SH-SY5Y and CHLA-119 purchased from the Cell Bank of the Chinese Academy of Sciences Shanghai Institute of Cell Biology (Shanghai, China) were maintained in a 1:1 mixture of F12 and Dulbecco's modified Eagle's medium (DMEM) (HyClone/Thermo Fisher Scientific, Beijing, China) supplemented with $10 \%$ heat-inactivated fetal FBS (Hangzhou Sijiqing Biological Engineering Materials Co., Ltd, Hangzhou, China) in a humidified atmosphere of $5 \%$ of $\mathrm{CO}_{2}$ in air at $37^{\circ} \mathrm{C}$. ABT-263 was purchased from Biochempartner (Shanghai, China) and was dissolved in DMSO with a stock concentration of $100 \mathrm{mM}$ and stored at $-20^{\circ} \mathrm{C}$. NCTD was purchased from Shaanxi Huike Plants Exploitation (Xi'an, China), and the purity was $>98 \%$ as determined by high-performance liquid, and was dissolved in DMEM with a stock concentration of $1 \mathrm{mM}$ and stored at $-20^{\circ} \mathrm{C}$.

MTT viability assay. Cell viability was measured by 3-[4,5-dimethylthiazol-2-thiazolyl]-2,5-diphenyl-tetrazolium bromide (MTT) assay. The percentages of absorbance relative to those of untreated control samples were plotted as a linear function of drug concentration. Inhibition of cell viability was measured by percentage of viable cells relative to the control: $\%$ Inhibition $=100 \% \mathrm{x}$ ODT/ODC, where ODT is the average OD value of the treated samples and ODC is the average OD value of the control samples.

Cell death and flow cytometry apoptosis assays. Cell death was quantitated by microscopic examination in trypan blue exclusion assays. Blue-stained and considerably shrunken cells were counted as non-viable cells. SubG1 apoptosis in cells was examined by propidium iodide (PI; $50 \mu \mathrm{g} / \mathrm{ml}$ in PBS) staining in the presence of RNase A $(100 \mu \mathrm{g} / \mathrm{ml})$ and flow cytometry with a BD LSR II system (BD Biosciences, Shanghai, China).

Apoptosis TUNEL assay. Apoptosis terminal deoxynucleotidyl transferase dUTP nick end labeling (TUNEL) assay was performed with a commercial kit of DeadEnd ${ }^{\mathrm{TM}}$ Colorimetric TUNEL assay from Promega (Shanghai, China).

Clonogenic assay. For clonogenic assay, 1,000 cells were seeded into 6 -well dishes in $3 \mathrm{ml}$ of medium. The next day, cells were treated as indicated, and then maintained for 14 days at $37^{\circ} \mathrm{C}$ in a $5 \% \mathrm{CO}_{2}$ incubator. Cells were washed with drug-free medium, stained with $0.05 \%$ methylene blue, and cell colonies ( $>50$ cells) were counted at 14 days. Assays were performed in duplicate with at least three independent repetitions per treatment.

Western blot analysis and cell fractionation. Western blot analyses and cell fractionation were performed as previously described (21). Antibodies used were: anti-cytochrome $c$ rabbit polyclonal antibody (556432) from BD Biosciences (Shanghai, China); anti-actin goat polyclonal antibody, and HRP-conjugated secondary anti-mouse, anti-goat and antirabbit antibodies from Santa Cruz Biotechnology (Shanghai, China); anti-caspase-9 rabbit polyclonal antibody (9502), anticaspase-3 rabbit polyclonal antibody (9662), anti-Bcl-2 rabbit polyclonal antibody (2870), anti-Mcl-1 rabbit polyclonal antibody (4572) and anti-COX IV from Cell Signaling Technology (Shanghai, China). Protein expression levels were quantified by densitometry (ImageJ program). The relative density was calculated as follows: Relative density = (band density of the sample)/(band density of the control).

RNA interference. The siRNA transfections were carried out using Lipofectamine RNAiMax transfection reagent (Invitrogen) according to the manufacturer's instructions. Validated siRNA for Mcl-1 and Noxa duplexes were purchased from Invitrogen (Shanghai, China). A non-silencing control siRNA (siCTL) was used as the control. Concentration for transfection was $10 \mathrm{nmol} / \mathrm{l}$ of each siRNA.

Statistical analysis. Statistical analyses were performed by one-way ANOVA using SPSS (version 13.0; SPSS Inc, Chicago, IL, USA). $\mathrm{P}<0.05$ was considered statistically significant, $\mathrm{P}<0.01$ was considered highly statistically significant.

\section{Results}

ABT-263 is ineffective in neuroblastoma SH-SY5Y and CHLA-119 cell lines. We initiated our study to evaluate the activity of Bcl-2 inhibitor ABT-263 in SH-SY5Y and CHLA-119 cell lines, since these two cell lines expressed a high level of Bcl-2 $(4,6)$. MTT cell proliferation assays showed that treatment with ABT-263 for $24 \mathrm{~h}$ achieved $\mathrm{IC}_{50}$ values of 70 and $98 \mu \mathrm{M}$, respectively, in SH-SY5Y and CHLA-119 cell lines. ABT-263 at $10 \mu \mathrm{M}$ inhibited cell proliferation only by 7 and 4\%, respectively, in the two cell lines (Fig. 1A). Treatment with ABT-263 for a longer time (48 and $72 \mathrm{~h}$ ) did not appreciably increase the inhibitory effect on cell proliferation. Consistently, trypan blue staining assays showed 

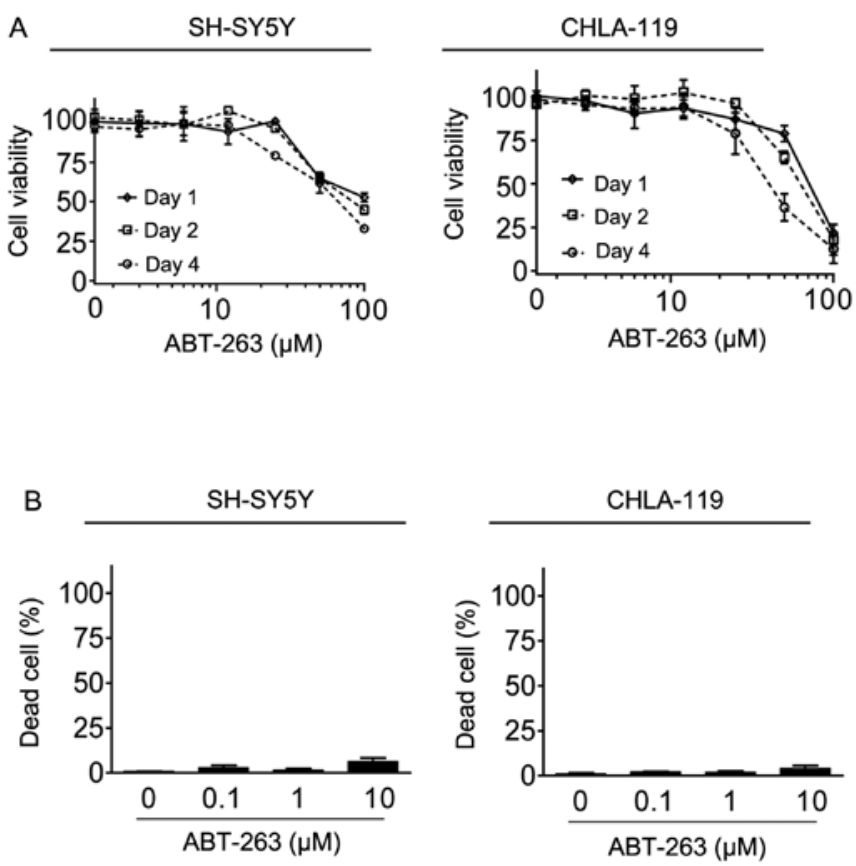

Figure 1. Neuroblastoma SH-SY5Y and CHLA-119 cell lines are resistant to ABT-263. (A) SH-SY5Y and CHLA-119 cell lines were treated with ABT-263 as indicated. Cell viability was examined with MTT assays. (B) SH-SY5Y and CHLA-119 cell lines were treated with ABT-263 for $48 \mathrm{~h}$. Cell death was examined with trypan blue exclusion assays.

that treatment with ABT-263 at $10 \mu \mathrm{M}$ for $48 \mathrm{~h}$ only induced approximately $6 \%$ and $7 \%$ cell death, respectively, in the two cell lines (Fig. 1B). These results showed that ABT-263 is ineffective in these neuroblastoma cell lines.

NCTD markedly increases Noxa expression in neuroblastoma cells. A number of previous studies have reported that NCTD could modulate the levels of Bcl-2 family proteins in oral cancer, liver cancer and breast cancer cells (20-22). We next investigated whether this drug had a similar effect on neuroblastoma cells. MTT assays were used to determine a subtoxic concentration range of NCTD in the cell lines. We found that NCTD at 15-60 $\mu \mathrm{M}$ dose-dependently and partially inhibited cell viability in both cell lines (Fig. 2A). Thus, we treated SH-SY5Y and CHLA-119 cell lines with these sub-toxic concentrations of NCTD for $48 \mathrm{~h}$ (Fig. 1B), and then examined the expression of several key members of Bcl-2 family proteins in cells. Western blot analysis showed that NCTD at these concentrations did not have an effect on the expressions of Bad and Bax, Bak, and had a modest inhibitory effect on the expressions of Mcl-1 and Bcl-2 in the two cell lines (Fig. 1B). Contrarily, the same treatments markedly increased the level of Noxa in the two cell lines in a dose-dependent manner. Densitometry analysis of western blotting results showed NCTD at 15,30 and $60 \mu \mathrm{M}$ increased the level of Noxa 1.6-, 3.8- and 5.7-fold in the SH-SY5Y cell line respectively, and increased the level of Noxa 2.2-, 4.3- and 4.4-fold, in the CHLA-119 cell line respectively (Fig. 1B).

NCTD enhances ABT-263-mediated apoptosis in neuroblastoma cells. We next investigated whether NCTD enhanced ABT-263-mediated apoptosis induction in neuroblastoma
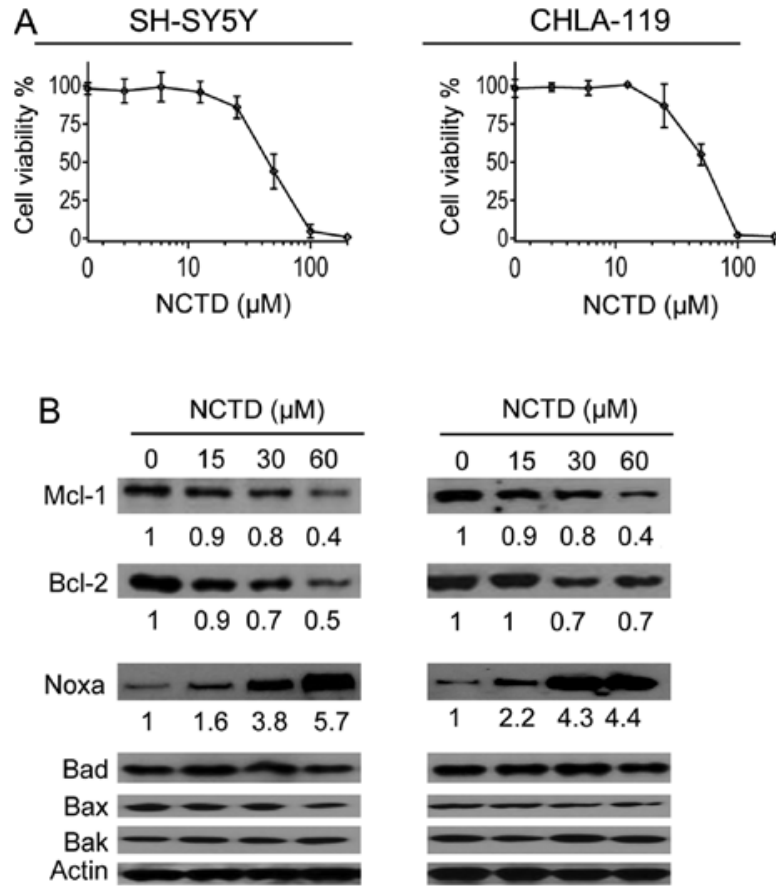

Figure 2. NCTD increases Noxa expression in neuroblastoma cells (A) SH-SY5Y and CHLA-119 cell lines were treated with NCTD as indicated for $48 \mathrm{~h}$. Cell viability was examined with MTT assays. (B) SH-SY5Y and CHLA-119 cell lines were treated with subtoxic concentrations of NCTD as indicated for $48 \mathrm{~h}$. Cells were harvested and lysed, whole-cell lysates were analyzed by western blotting for expressions of Mcl-1, Bcl-2, Noxa, Bad and Bax, Bak with specific antibodies. Actin was used as a loading control.

cells. We treated neuroblastoma cells with NCTD at $30 \mu \mathrm{M}$ alone, ABT-263 at $2 \mu \mathrm{M}$ alone or their combination for $24 \mathrm{~h}$, and performed PI staining and flow cytometry to quantify the percentage of apoptotic cells in the "sub-G1" peak. We observed that single-agent treatments induced apoptosis only in $<10 \%$ of neuroblastoma cells (Fig. 3A). In contrast, their combination induced $68 \%$ and $65 \%$ of cells undergoing apoptosis in SH-SY5Y and CHLA-119 cell lines, respectively (Fig. 3A). We then performed TUNEL assay in SH-SY5Y cell line to validate the results of flow cytometry assays. We found that treatment with either agent alone induced only a very small fraction of cells positively stained with TUNEL, whereas their combination not only resulted in TUNEL positive in most cells, but also led to cells shrinking into a dense, round mass, a distinct morphological characteristic of apoptosis (Fig. 3B) (14). These results demonstrated that NCTD potently enhanced ABT-263-triggered apoptosis in neuroblastoma cells.

NCTD enhances ABT-263-mediated inhibition of cell proliferation and clonal formation in neuroblastoma cells. Subsequently, we investigated whether NCTD could enhance ABT-263-mediated inhibition of cell proliferation in neuroblastoma cells. We treated the cells with ABT-263 alone or in combination with NCTD for 4 days, then examined cell proliferation with MTT assays. We found that NCTD dose-dependently enhanced ABT-263-mediated cell proliferation inhibition in the two cell lines (Fig. 4A). Notably, the enhancement of proliferation inhibition correlated well with upregulation of Noxa by NCTD in the two cell lines (Figs. 2 
A
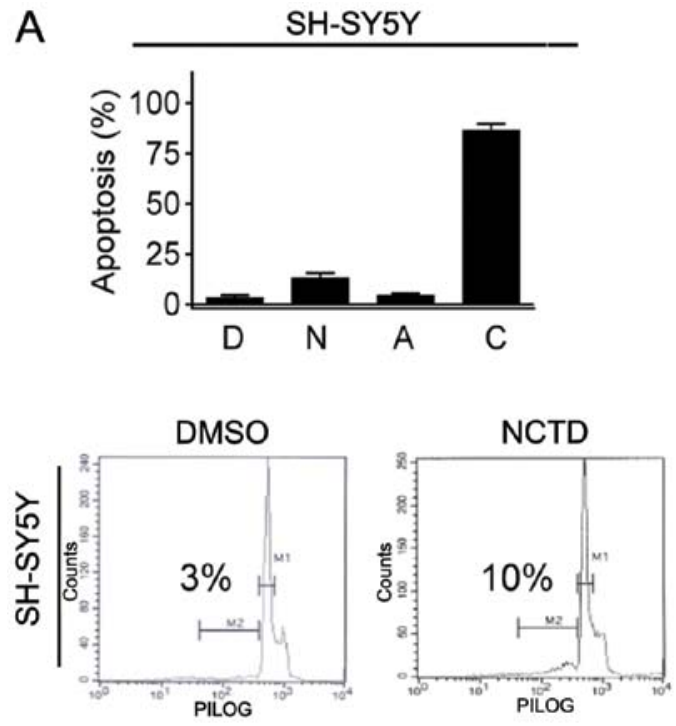
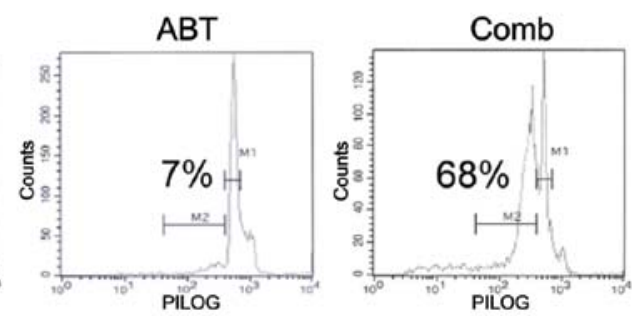

B
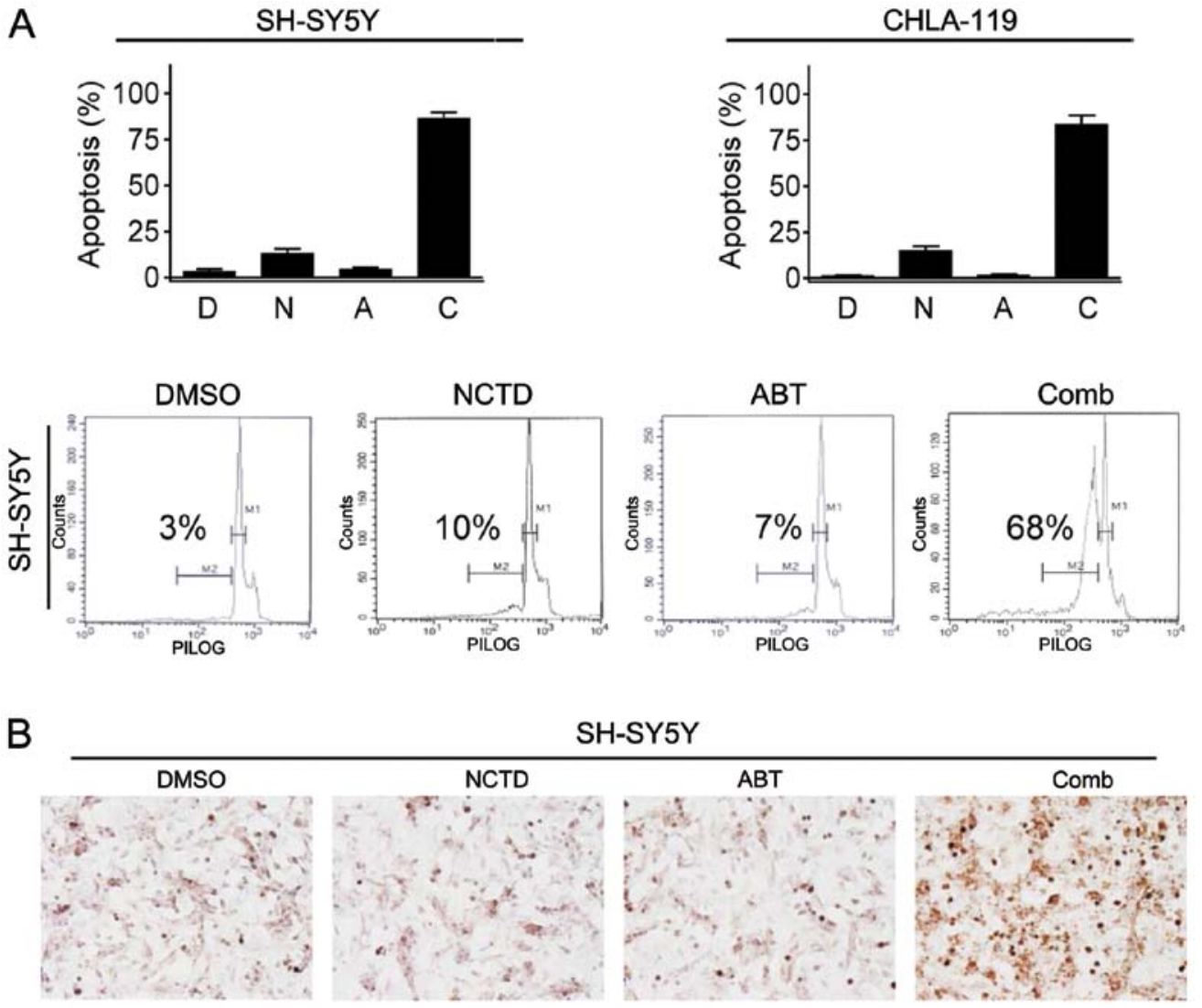

Figure 3. NCTD enhances ABT-263-triggered apoptosis in neuroblastoma cells. (A) SH-SY5Y and CHLA-119 cell lines were treated with ABT-263 at $2 \mu$ M alone, NCTD at $30 \mu \mathrm{M}$ alone or their combination for $24 \mathrm{~h}$. Cells were harvested and stained with PI. Percentage of cells in the "sub-G1 peak" was quantified with flow cytometry assay (top panels). Data of PI-positive cells show means \pm SD of three experiments. ${ }^{* *} \mathrm{P}<0.01$ (lower panel). Representative figures of sub-G1 analysis for SH-SY5Y cell line. DMSO was used as mock control treatment. (B) SH-SY5Y cell line was treated with ABT-263 at $2 \mu$ M alone, NCTD at $30 \mu \mathrm{M}$ alone or their combination on glass slides. After treatment for $24 \mathrm{~h}$, cells were examined with a TUNEL assay, and images were captured.

and 4A). For instance, in the SH-SY5Y cell line, NCTD at $15 \mu \mathrm{M}$, a concentration increasing the level of Noxa 1.6-fold only reduced the $\mathrm{IC}_{50}$ value by $45 \%$ (from 40 to $22 \mu \mathrm{M}$ ), while NCTD at 30 and $60 \mu \mathrm{M}$, concentrations increasing the level of Noxa 3.8-fold and 5.7-fold, in combination with ABT-263 achieved $100 \%$ cell viability inhibition in the two neuroblastoma cell lines.

Neuroblastoma cells were grown in the presence of NCTD alone, ABT-263 alone or both for 2 weeks. Clonogenic assays were performed to determine whether the combination of NCTD and ABT-263 had long-term stronger anticancer activity in SH-SY5Y and CHLA-1 cell lines. As shown in Fig. 4, ABT-263 alone showed no efficacy and NCTD at $30 \mu \mathrm{M}$ showed modest effect, whereas the combination achieved a significant improvement in the inhibition of colony formation (combination vs. ABT-263, P<0.001; combination vs. NCTD, $\mathrm{P}<0.01)$.

NCTD enhances ABT-263-triggered release of cytochrome $c$ and activation of caspases in neuroblastoma cells. Cytochrome $c$ released from the mitochondrial intermembrane space into the cytosol is a crucial step in ABT-263-triggered apoptosis $(10,11,23)$. We next investigated whether treatment with ABT-263 alone, NCTD alone or both could induce cytosolic release of cytochrome $c$ in neuroblastoma cells. As shown in Fig. 5A, treatment with either NCTD or ABT-263 alone induced a negligible release of cytochrome $c$. By sharp contrast, co-treatment with the two drugs resulted in a robust increase of cytochrome $c$ release in the two cell lines, which was accompanied by a modest decrease of cytochrome $c$ in mitochondria-enriched membrane fraction (Fig. 5B). Hence, these data indicated that NCTD markedly enhanced ABT-263-triggered damage of the mitochondrial outer membrane integrity and allowed the release of the proapoptotic protein cytochrome $c$ into the cytosol in the neuroblastoma cells.

Activation of caspases is another key event following cytochrome $c$ release in apoptosis signaling (24). We then treated the cells with single agents or combination, and examined whether caspase-9, -3 were activated in neuroblastoma cells with western blot analysis. We found that as compared to the minimal effect by single-agent treatments, combination treatment with ABT-263 and NCTD for $24 \mathrm{~h}$ led to a robust accumulation of cleaved fragments of initiator caspase- 9 in the two cell lines. Similarly, substantial accumulation of cleaved effector caspase-3 was observed in the combination treatment but not in single-agent treatments. We also observed that PARP, a substrate of caspase-3, was cleaved upon treatment with the combination, but not by treatment with single agents (Fig. 5C).

To determine whether caspases were required for the combination effect of NCTD and ABT-263, SH-SY5Y and CHLA-119 cell lines were pretreated with the $50 \mu \mathrm{M}$ of 
A

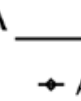

SH-SY5Y

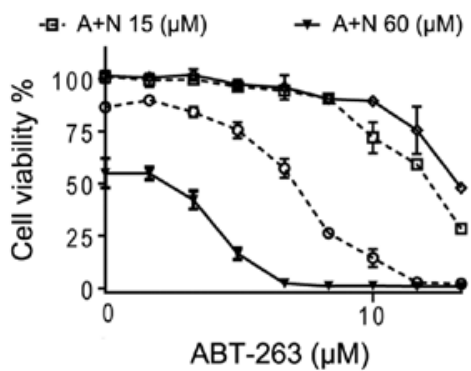

B

充

C

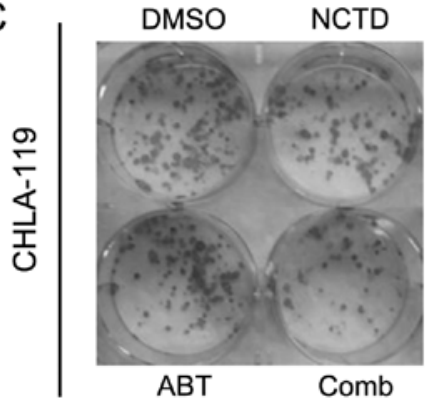

CHLA-119
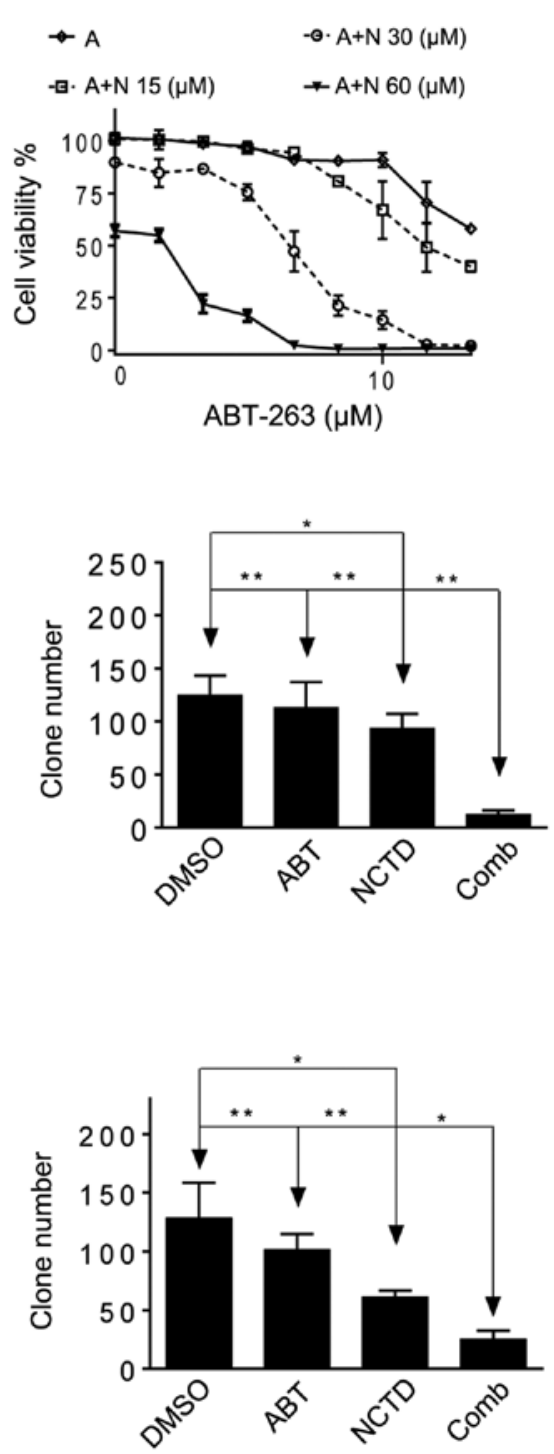

Figure 4. NCTD enhances ABT-263-mediated anticancer activity in neuroblastoma cells. (A) SH-SY5Y and CHLA-119 cell lines were treated with ABT-263 (A) at $2 \mu \mathrm{M}$ alone or in combination with NCTD (N) as indicated for $48 \mathrm{~h}$. Cell viability was examined with MTT assays. (B) SH-SY5Y and (C) CHLA-119 cell lines were treated with ABT-263 at $2 \mu \mathrm{M}$ (ABT) or NCTD at $30 \mu \mathrm{M}$ alone or both (Comb) as indicated for 14 days. Clonogenicity was examined as described in Materials and methods. Representative dishes from the clonogenicity assay (left panels). The results shown here are representative of three independent experiments (right panels).

pancaspase inhibitor (zVAD.fmk) for $1 \mathrm{~h}$ before the addition of NCTD $(30 \mu \mathrm{M})$ and ABT-263 $(2 \mu \mathrm{M})$ (Fig. 5D). Inhibition of caspase activity significantly attenuated the cell death induction by the combination, evidently showing that a caspase-dependent mechanism was involved in the anticancer activity of the two drugs.

Collectively, these results demonstrate that NCTD greatly enhances ABT-263-triggered apoptosis in neuroblastoma cells.

Noxa plays an essential role in the enhancement of ABT-263mediated cell death by NCTD in neuroblastoma cells. Mcl-1 has been identified as the key mediator of ABT-263-resistance in cancer cells $(10,11,14,15)$. Given the fact that Noxa is an endogenous inhibitor of Mcl-1 (25-27), we hypothesized that upregulation of Noxa might play a role in the enhancement of ABT-263-mediated anticancer activity by NCTD. To inves- tigate this hypothesis, we knocked down Noxa with specific siRNAs in the two neuroblastoma cell lines. As shown in Fig. 6, transfection for $48 \mathrm{~h}$ effectively suppressed Noxa expression in SH-SY5Y and CHLA-119 cell lines, but had no effect on the expression of Mcl-1 in the two cell lines. MTT assays showed that inhibition of Noxa expression by siRNA significantly attenuated cell viability inhibition mediated by ABT-263 in combination with NCTD. For instance, ABT-263 in combination with NCTD at $30 \mu \mathrm{M}$ induced $82 \%$ cell death in siCTL transfected SH-SY5Y cells, while the same treatments only caused $32 \%$ cell death in siNoxa transfected cells $(\mathrm{P}<0.01)$ (Fig. 6). An inhibitory effect on the combination by siNoxa was also observed in the CHLA-119 cell line. These results indicate that upregulation of Noxa is required in the enhancement of ABT-263-mediated anticancer activity by NCTD in neuroblastoma cells. 
A

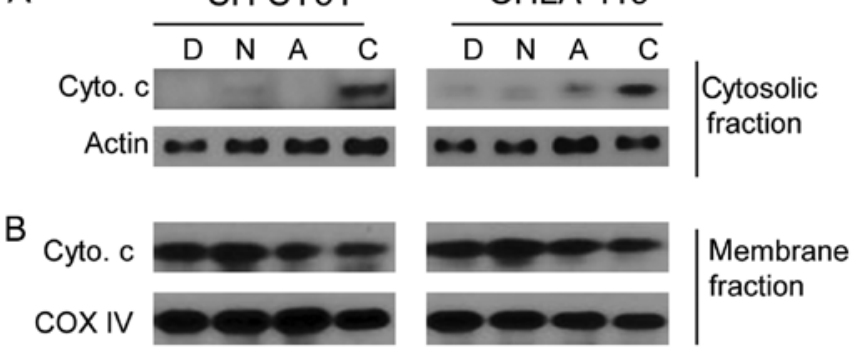

$\mathrm{C}$

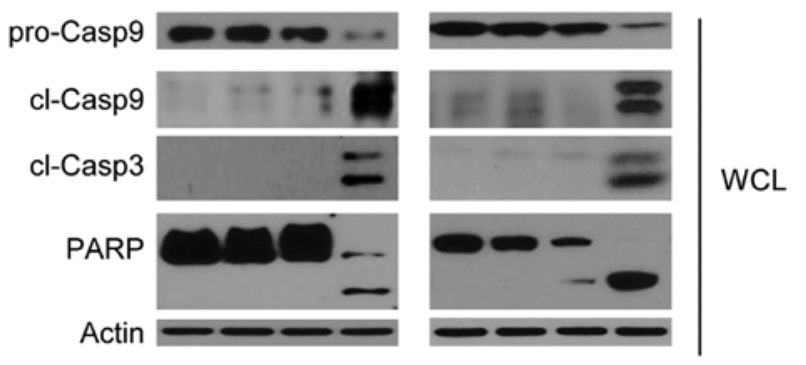

D

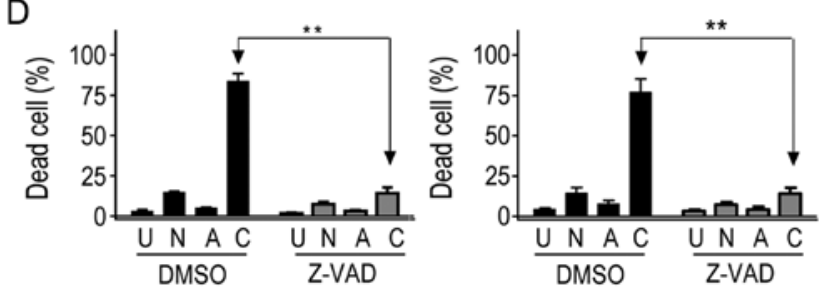

Figure 5. NCTD enhances ABT-263-triggered release of cytochrome $c$ and caspase activation in neuroblastoma cells. (A and B) SH-SY5Y and CHLA-119 cell lines were treated with ABT-263 (A) at $2 \mu \mathrm{M}$ alone, NCTD (N) at $30 \mu \mathrm{M}$ alone, their combination (C) for $48 \mathrm{~h}$ and cells were harvested. (A and B) Cells were fractioned. Expression of cytochrome $c$ in cytoplasm (A) and in membrane enriched fraction (B) was examined by western blotting and specific antibody. Actin and COX IV were used as loading controls. (C) The expressions of caspases and PARP in whole-cell lysates were examined by western blotting. Actin was used as a loading control. (D) SH-SY5Y and CHLA-119 cell lines were pretreated with $50 \mu \mathrm{M}$ of pancaspase inhibitor (zVAD.fmk) or DMSO for $1 \mathrm{~h}$ before the addition of ABT-263 (A) at $2 \mu \mathrm{M}$ alone, NCTD (N) at $30 \mu \mathrm{M}$ alone, their combination (C) for $48 \mathrm{~h}$, and cell death induction was examined with trypan blue exclusion assays. ${ }^{* *} \mathrm{P}<0.01$.

\section{Discussion}

ABT-737 and ABT-263 are small molecule Bcl-2 inhibitors developed as novel anticancer drugs. Preclinical studies demonstrated that these two drugs exhibit promising anticancer efficacy in certain types of cancers. Therefore, ABT-737 and ABT-263 represent promising anticancer therapies. Nonetheless, due to low binding affinity to another member of antiapoptotic Bcl-2 protein, Mcl-1, ABT-737 and ABT-263 have very weak anticancer activity in a large number of other cancer types, such as neuroblastoma, which express an elevated level of Mcl-1. The ineffectiveness of ABT-737 and ABT-263 in those cancers poses a key problem for their potential application in a wide range of cancer types (14-16). Accordingly, finding approaches to overcome ABT-263/ABT-737-resistance through inhibition of Mcl-1, directly or indirectly, is urgently needed.
A

\section{SH-SY5Y}
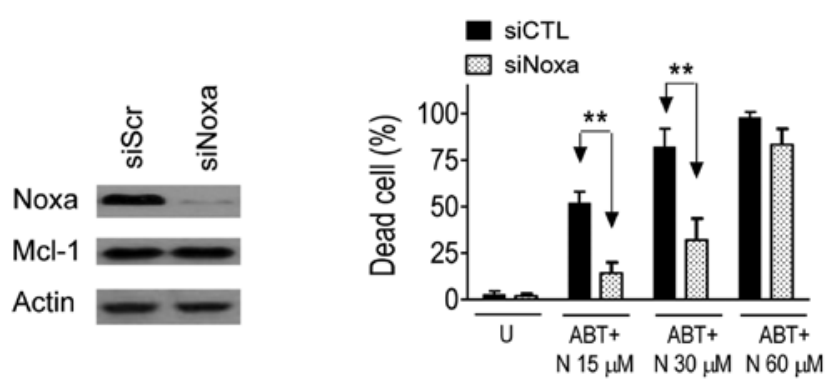

B CHLA-119

Figure 6. Noxa plays a critical role in the enhancement of ABT-263-activity by NCTD in neuroblastoma cells. (A) SH-SY5Y and (B) CHLA-119 cell lines were transfected with siCTL or siNoxa for $48 \mathrm{~h}$. Expressions of Noxa were analyzed by western blotting. Actin was used as a loading control (left panels). Cells were transfected with siCTL or siNoxa for $24 \mathrm{~h}$. Then, cells were treated with ABT-263 at $2 \mu \mathrm{M}$ plus NCTD at 15,30 and $60 \mu \mathrm{M}$ for $48 \mathrm{~h}$. Cell death was examined with trypan blue exclusion assays (right panels).

The strategies that directly target Mcl-1 expression have been investigated extensively. For instance, cyclin-dependent kinase (CDK) inhibitors, such as flavopiridol and roscovitine, have been found to enhance ABT-737 activity by suppressing Mcl-1 expression in several types of cancer (15). Meanwhile, an alternative strategy that sensitizes cancer cells to ABT-263/ABT-737-mediated anticancer activity is the use of agents capable of increasing Noxa expression. Noxa belongs to the proapoptotic $\mathrm{Bcl}-2 \mathrm{BH} 3$ subfamily, and is known as an endogenous Mcl-1 inhibitor (25-27). Increasing Noxa expression has been reported to enhance ABT-263-activity in a number of cancer types. For instance, Simonin et al found that platinum compounds enhance ABT-737-mediated apoptosis in ovarian carcinoma cells by upregulation of Noxa expression (27). Zinn et al reported that chloroquine overcome ABT-737-resistance in small lung cancer cells by increasing Noxa expression (28). In the current study, we observed that NCTD considerably enhanced ABT-263mediated anticancer activity, which was accompanied by marked increase of Noxa expression in neuroblastoma cells. By employing specific siRNA, we confirmed that Noxa played an essential role in the enhancement of ABT-263mediated anticancer activity by NCTD. Since NCTD is a conventional anticancer drug, and ABT-263 is being tested in clinic, our study has more translational significance in ABT-based cancer therapy. 
NCTD is a demethylated analog of cantharidin, the major bioactive constituent of Chinese blister beetle Mylabris, which has been used in China to treat tumors, inflammation and many other conditions for a long time. Previous studies showed that NCTD could modulate the expression of Bcl-2 and Mcl-1 in oral, breast and hepatocellular cancer cells $(19-22,29)$. Here, we explored whether this drug also inhibited antiapoptotic Bcl-2 proteins in neuroblastoma cells. However, we observed that NCTD at the applied concentrations only had a modest effect on downregulation of Mcl-1 and Bcl-2. By contrast, NCTD could markedly increase the expression of Noxa in the two neuroblastoma cell lines. These discrepancies between this study and previous reports may reflect the diverse response of different cancer types to NCTD, and may also indicate the need for elucidation of the true mechanism by which NCTD elicits anticancer activity in the future.

Several pieces of evidence indicated that combination treatment by two drugs triggered activation of the mitochondrial apoptosis signaling pathway. Firstly, flow cytometery and TUNEL staining assays showed that co-treatment with ABT-263 and NCTD induced apoptosis in a large fraction of cells. Secondly, western blot analysis showed that combination treatment induced activation of several typical biomarkers of mitochondria-mediated apoptosis, including cytosolic cytochrome $c$ release, activation of initiator caspase- 9 and effector caspase-3 and PARP cleavage. Thirdly, cell killing induced by the combination could be largely rescued by a pancaspase inhibitor zVAD.fmk. Once again, our findings support that the combinational effect of ABT-737/ABT-263 with other approaches is dependent on ABT-737/ABT-263-triggered mitochondrial apoptosis signaling (15-17,21).

\section{Acknowledgements}

This research was supported by the National Natural Science Foundation of China (81101685).

\section{References}

1. Meadows A, Baum E, Fossati-Bellani F, et al: Second malignant neoplasms in children: an update from the late effects study group. J Clin Oncol 3: 532-538, 1985.

2. Welch C, Chen Y and Stallings R: MicroRNA-34a functions as a potential tumor suppressor by inducing apoptosis in neuroblastoma cells. Oncogene 26: 5017-5022, 2007.

3. George R, Sanda T, Hanna M, et al: Activating mutations in ALK provide a therapeutic target in neuroblastoma. Nature 455 975-978, 2008

4. Goldsmith K, Lestini B, Gross M, et al: BH3 response profiles from neuroblastoma mitochondria predict activity of small molecule Bcl-2 family antagonists. Cell Death Differ 17: $872-882,2010$

5. Kroemer G: The proto-oncogene $\mathrm{Bcl}-2$ and its role in regulating apoptosis. Nature Med 3: 614-620, 1997.

6. Fang H, Harned T, Kalous O, Maldonado V, DeClerck Y and Reynolds C: Synergistic activity of fenretinide and the Bcl-2 family protein inhibitor ABT-737 against human neuroblastoma. Clin Cancer Res 17: 7093-7104, 2011.

7. Castle V, Heidelberger K, Bromberg J, Ou X, Dole M and Nuñez G: Expression of the apoptosis-suppressing protein bcl-2, in neuroblastoma is associated with unfavorable histology and N-myc amplification. Am J Pathol 143: 1543-1550, 1993.
8. Dole M, Nuñez G, Merchant A, Maybaum J, Rode C, Bloch C and Castle V: Bcl-2 inhibits chemotherapy-induced apoptosis in neuroblastoma. Cancer Res 54: 3253-3259, 1994

9. Hanada M, Krajewski S, Tanaka S, et al: Regulation of Bcl-2 oncoprotein levels with differentiation of human neuroblastoma cells. Cancer Res 53: 4978-4986, 1993.

10. Tse C, Shoemaker A, Adickes J, et al: ABT-263: a potent and orally bioavailable Bcl-2 family inhibitor. Cancer Res 68: 3421-3428, 2008.

11. Oltersdorf T, Elmore S, Shoemaker A, et al: An inhibitor of Bcl-2 family proteins induces regression of solid tumours. Nature 435: 677-681, 2005.

12. Gandhi L, Camidge D, Ribeiro de Oliveira M, et al: Phase I study of Navitoclax (ABT-263), a novel Bcl-2 family inhibitor, in patients with small-cell lung cancer and other solid tumors. J Clin Oncol 29: 909-916, 2011.

13. Rudin C, Hann C, Garon E, et al: Phase II study of single-agent navitoclax (ABT-263) and biomarker correlates in patients with relapsed small cell lung cancer. Clin Cancer Res 18: 3163-3169, 2012.

14. Konopleva M, Contractor R, Tsao T, et al: Mechanisms of apoptosis sensitivity and resistance to the BH3 mimetic ABT-737 in acute myeloid leukemia. Cancer Cell 10: 375-388, 2006.

15. Billard C: BH3 mimetics: status of the field and new developments. Mol Cancer Ther 12: 1691-1700, 2013.

16. Goldsmith K, Gross M, Peirce S, et al: Mitochondrial Bcl-2 family dynamics define therapy response and resistance in neuroblastoma. Cancer Res 72: 2565-2577, 2012.

17. Klymenko T, Brandenburg M, Morrow C, Dive C, Makin G: The novel Bcl-2 inhibitor ABT-737 is more effective in hypoxia and is able to reverse hypoxia-induced drug resistance in neuroblastoma cells. Mol Cancer Ther 10: 2373-2383, 2011.

18. Wang G: Medical uses of mylabris in ancient China and recent studies. J Ethnopharmacol 26: 147-162, 1989.

19. Yang E, Tang W,Zhang K, Cheng L and Mack PO: Norcantharidin inhibits growth of human HepG2 cell-transplanted tumor in nude mice and prolongs host survival. Cancer Lett 117: 93-98, 1997.

20. Kok SH, Cheng SJ, Hong CY, et al: Norcantharidin-induced apoptosis in oral cancer cells is associated with an increase of proapoptotic to antiapoptotic protein ratio. Cancer Lett 217: 43-52, 2005.

21. Zhang S, Li G, Ma X, et al: Norcantharidin enhances ABT-737induced apoptosis in hepatocellular carcinoma cells by transcriptional repression of Mcl-1. Cell Signal 24: 1803-1809, 2012.

22. Huang Y, Liu Q, Liu K, Yagasaki K, and Zhang G: Suppression of growth of highly-metastatic human breast cancer cells by norcantharidin and its mechanisms of action. Cytotechnology 59: 201-208, 2009.

23. Yang J, Liu X, Bhalla K et al: Prevention of apoptosis by Bcl-2: release of cytochrome $\mathrm{c}$ from mitochondria blocked. Science 275 : 1129-1132, 1997.

24. Green DR and Kroemer G: The pathophysiology of mitochondrial cell death. Science 305: 626-629, 2004.

25. Mazumder S, Choudhary G, Al-Harbi S, Almasan A: Mcl-1 phosphorylation defines ABT-737 resistance that can be overcome by increased NOXA expression in leukemic B cells. Cancer Res 72: 3069-3079, 2012.

26. Okumura K, Huang S and Sinicrope F: Induction of Noxa sensitizes human colorectal cancer cells expressing Mcl-1 to the small-molecule Bcl-2/Bcl-xL inhibitor, ABT-737. Clin Cancer Res 14: 8132-8142, 2008.

27. Simonin K, N'Diaye M, Lheureux S, et al: Platinum compounds sensitize ovarian carcinoma cells to ABT-737 by modulation of the Mcl-1/Noxa axis. Apoptosis 18: 492-508, 2013.

28. Zinn R, Gardner E, Dobromilskaya I, et al: Combination treatment with ABT-737 and chloroquine in preclinical models of small cell lung cancer. Mol Cancer 12: Mar 2, 2013. doi: 10.1186/1476-4598-12-16.

29. Yang PY, Chen MF, Kao YH, et al: Norcantharidin induces apoptosis of breast cancer cells: involvement of activities of mitogen activated protein kinases and signal transducers and activators of transcription. Toxicol In Vitro 25: 699-707, 2011. 